Pacific

Journal of

Mathematics

\title{
FLAT FRONTS IN HYPERBOLIC 3-SPACE
}

Masatoshi KokUbu, Masaaki Umehara and Kotaro Yamada 


\title{
FLAT FRONTS IN HYPERBOLIC 3-SPACE
}

\author{
Masatoshi Kokubu, Masaaki Umehara and Kotaro Yamada
}

We investigate flat surfaces in hyperbolic 3-space with admissible singularities, called flat fronts. An Osserman-type inequality for complete flat fronts is shown. When equality holds in this inequality, we show that all the ends are embedded, and give new examples for which equality holds.

\section{Introduction}

It is a classical fact that any complete flat surface in the hyperbolic 3space $H^{3}$ must be a horosphere or a hyperbolic cylinder. However, this does not imply the lack of an interesting global theory for flat surfaces. Recently, Gálvez, Martínez and Milán [4] established a Weierstrass-type representation formula for such surfaces. More recently, the authors [7] proved another representation formula constructing a flat surface from a given pair of hyperbolic Gauss maps, and also gave new examples.

In this paper, we investigate global properties of flat surfaces with admissible singularities, accounting for all the previous examples in [4] and [7]. (A singular (i.e., degenerate) point is called admissible if the corresponding points on nearby parallel surfaces are regularly immersed. See Section 2.) Such surfaces are characterized as the projections of Legendrian immersions in the unit cotangent bundle $T_{1}^{*} H^{3}$ of $H^{3}$, called flat fronts. The 5-manifold $T_{1}^{*} H^{3}$ has the canonical contact form $\eta$. If we identify $H^{3}$ with the Poincaré ball $\left(D^{3} ; x^{1}, x^{2}, x^{3}\right)$, any element $\alpha$ of the cotangent bundle $T^{*} H^{3}$ can be written as

$$
\alpha=p_{1}(\alpha) d x^{1}+p_{2}(\alpha) d x^{2}+p_{3}(\alpha) d x^{3} \quad\left(\in T^{*} H^{3}\right) .
$$

Then $\left(p_{1}, p_{2}, p_{3}, x^{1}, x^{2}, x^{3}\right)$ gives a canonical coordinate system of $T^{*} H^{3}$ and the canonical form on $T^{*} H^{3}$,

$$
\eta=p_{1} d x^{1}+p_{2} d x^{2}+p_{3} d x^{3}
$$

which induces a canonical contact form on $T_{1}^{*} H^{3}$. An immersion $L: M^{2} \rightarrow$ $T_{1}^{*} H^{3}$ is called Legendrian if the pullback $L^{*} \eta$ vanishes identically. For a given immersion $f: M^{2} \rightarrow H^{3}$, there exists a unique Legendrian immersion

$$
L_{f}: M^{2} \longrightarrow T_{1}^{*} H^{3}
$$




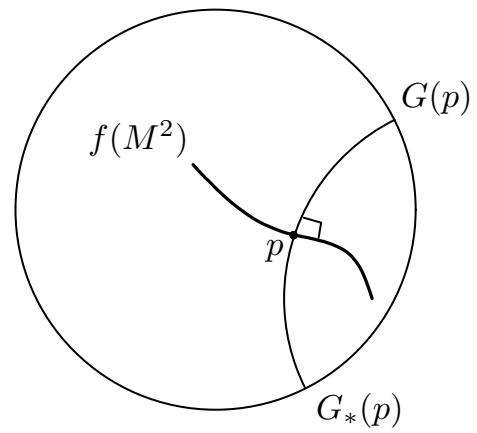

Figure 1. Hyperbolic Gauss maps.

such that $\pi \circ L_{f}=f$, where $\pi: T_{1}^{*} H^{3} \rightarrow H^{3}$ is the projection. That is, any immersion can be lifted to a Legendrian immersion. However, the converse is not true. A projection

$$
\pi \circ L: M^{2} \longrightarrow H^{3}
$$

of a Legendrian immersion $L$ is called a (wave) front, which may have singular points (points where the Jacobi matrix degenerates.) A point which is not singular is called regular, where the first fundamental form is positive definite. The Gaussian curvature is well-defined at regular points. A front is called flat if the Gaussian curvature vanishes at each regular point.

A front $f$ is called complete if there is a symmetric tensor $T$ on $M^{2}$ which has compact support such that $T+d s^{2}$ is a complete Riemannian metric on $M^{2}$, where $d s^{2}$ is the first fundamental form of $f$. If $M^{2}$ is orientable, $M^{2}$ can be regarded as a Riemann surface whose complex structure is compatible with respect to the pullback of the Sasakian metric on $T_{1}^{*} H^{3}$ by $L_{f}$. Moreover, the second fundamental form is Hermitian with respect to this structure, and there is a closed Riemann surface $\bar{M}^{2}$ such that $M^{2}$ is biholomorphic to $\bar{M}^{2} \backslash\left\{p_{1}, \ldots, p_{n}\right\}$. The points $p_{1}, \ldots, p_{n}$ are called the ends of $f$.

For each point $p \in M^{2}$, there exists a pair $\left(G(p), G_{*}(p)\right) \in S^{2} \times S^{2}$ of distinct points on the ideal boundary $S^{2}=\partial H^{3}$ such that the geodesic in $H^{3}$ starting from $G_{*}(p)$ towards $G(p)$ coincides with the oriented normal geodesic at $p$ (see Figure 1). The maps

$$
G, G_{*}: \bar{M}^{2} \backslash\left\{p_{1}, \ldots, p_{n}\right\} \longrightarrow S^{2}
$$

are called the positive and negative hyperbolic Gauss maps of $f$, respectively. They are holomorphic if we regard $S^{2}=\partial H^{3}$ as the Riemann sphere. An end $p_{j}$ is called regular if both $G$ and $G_{*}$ extend holomorphically across it. As we shall show later, there are many flat fronts with regular ends. Moreover, such surfaces satisfy the following global property: 
Theorem. An orientable complete flat front $f: \bar{M}^{2} \backslash\left\{p_{1}, \ldots, p_{n}\right\} \rightarrow H^{3}$ with regular ends satisfies the inequality

$$
\operatorname{deg} G+\operatorname{deg} G_{*} \geq n,
$$

where $\operatorname{deg} G$ is the degree of the holomorphic map $G: \bar{M}^{2} \rightarrow \mathbf{C P}^{1}=S^{2}$. Equality holds if and only if all ends are embedded.

This inequality is an analogue of the Osserman inequality

$$
2 \operatorname{deg} G+\chi\left(\bar{M}^{2} \backslash\left\{p_{1}, \ldots, p_{n}\right\}\right) \geq n,
$$

which holds for the Gauss map $G$ of either a complete minimal surface $f: \bar{M}^{2} \backslash\left\{p_{1}, \ldots, p_{n}\right\} \rightarrow \mathbf{R}^{3}$ with finite total curvature, or a surface $f: \bar{M}^{2} \backslash$ $\left\{p_{1}, \ldots, p_{n}\right\} \rightarrow H^{3}$ of mean curvature 1 . In these two cases, as in ours, equality implies the embeddedness of ends. (See $[\mathbf{8}, \mathbf{5}]$ for the minimal surface case and $[\mathbf{1 2}]$ for the hyperbolic case.)

To prove that equality implies the ends are embedded, a criterion for embeddedness of ends given in [4] will be applied. Furthermore, we shall classify flat 3-noids and exhibit a genus-1 flat front with regular ends (Section 4).

On the other hand, since the pullback of the Sasakian metric $d \sigma^{2}$ by the Legendrian lift of a complete flat front $f$ is complete, it satisfies the Cohn-Vossen inequality

$$
\frac{1}{2 \pi} \int_{M^{2}}\left(-K_{d \sigma^{2}}\right) d A_{d \sigma^{2}} \geq-\chi\left(M^{2}\right),
$$

where $d A_{d \sigma^{2}}$ is the area element of $d \sigma^{2}$ and $\chi\left(M^{2}\right)$ is the Euler number of $M^{2}$. In Section 3, we shall prove that equality holds if and only if all ends are asymptotic to a hyperbolic cylinder.

Note that flat hypersurfaces in $H^{n}(n \geq 4)$ are totally umbilic. So $n=3$ is the interesting case.

The authors are very grateful to P. Pirola and E. Musso for fruitful discussions. The authors also thank the referee for his valuable comments.

\section{Local properties of flat surfaces}

In this section, we review local properties of flat immersions. We denote by $H^{3}$ the hyperbolic 3-space of constant curvature -1. Let $M^{2}$ be a 2-manifold and

$$
f: M^{2} \longrightarrow H^{3}
$$

a flat immersion, meaning that the Gaussian curvature of the induced metric vanishes. It follows from the Gauss equation that the second fundamental form is positive or negative definite and thus $M^{2}$ is orientable. We fix an orientation of $M^{2}$. Then $M^{2}$ can be regarded as a Riemann surface such 
that the second fundamental form $d h^{2}$ is Hermitian. A holomorphic map or immersion

$$
E=\left(\begin{array}{ll}
A & B \\
C & D
\end{array}\right): M^{2} \longrightarrow \mathrm{SL}(2, \mathbf{C})
$$

is called a Legendrian curve or immersion if

$$
D d A-B d C=0 .
$$

Indeed, (1.1) implies the vanishing of the pullback of a holomorphic contact form on $\mathrm{SL}(2, \mathbf{C})$. As is shown in [4], there exists a holomorphic Legendrian immersion

$$
E_{f}: \widetilde{M}^{2} \longrightarrow \mathrm{SL}(2, \mathbf{C})
$$

defined on the universal cover $\widetilde{M}^{2}$ of $M^{2}$ such that $f$ is the projection of $E_{f}$ onto $H^{3}=\mathrm{SL}(2, \mathbf{C}) / \mathrm{SU}(2)$. $E_{f}$ is called a holomorphic Legendrian lift of $f$. Since (1.1) implies $E_{f}^{-1} d E_{f}$ is off-diagonal, we can set

$$
E_{f}^{-1} d E_{f}=\left(\begin{array}{cc}
0 & \theta \\
\omega & 0
\end{array}\right)
$$

The holomorphic 1-forms $\omega$ and $\theta$ are called the first canonical form and the second canonical form, respectively. We have

$$
\begin{aligned}
& \omega= \begin{cases}\frac{d A}{B} & (\text { if } d A \not \equiv 0 \text { or } B \not \equiv 0), \\
\frac{d C}{D} & (\text { if } d C \not \equiv 0 \text { or } D \not \equiv 0),\end{cases} \\
& \theta= \begin{cases}\frac{d B}{A} & (\text { if } d B \not \equiv 0 \text { or } A \not \equiv 0), \\
\frac{d D}{C} & (\text { if } d D \not \equiv 0 \text { or } C \not \equiv 0) .\end{cases}
\end{aligned}
$$

Here $\not \equiv 0$ means that the 1 -form or function in question are not identically zero.

In particular,

$$
\omega=\frac{d A}{B}=\frac{d C}{D} \quad \text { and } \quad \theta=\frac{d B}{A}=\frac{d D}{C}
$$

if all cases in (1.2) and (1.3) are well-defined. Then the first and second fundamental forms $d s^{2}$ and $d h^{2}$ have the expressions

$$
\begin{aligned}
& d s^{2}=(\omega+\bar{\theta})(\bar{\omega}+\theta)=\omega \theta+\bar{\omega} \bar{\theta}+|\omega|^{2}+|\theta|^{2}, \\
& d h^{2}=|\theta|^{2}-|\omega|^{2} .
\end{aligned}
$$


Though $\omega$ and $\theta$ are defined only on the universal cover $\widetilde{M}^{2}$, the first fundamental form $d s^{2}$ is well-defined on $M^{2}$, and then so is the (1,1)-part of $d s^{2}$ :

$$
d s_{1,1}^{2}:=|\omega|^{2}+|\theta|^{2} .
$$

Since (1.6) is well-defined on $M^{2}$, so are $|\omega|^{2}$ and $|\theta|^{2}$. Moreover, we can deduce that

$|\omega|^{2}$ and $|\theta|^{2}$ define flat pseudometrics on $M^{2}$ compatible with the complex structure of $M^{2}$.

The $(2,0)$-part of $d s^{2}$ is called the Hopf differential and is denoted by $Q$ :

$$
Q:=\omega \theta \text {. }
$$

The positive hyperbolic Gauss map $G$ and the negative hyperbolic Gauss map $G_{*}$ of the flat surface are defined by

$$
G=\frac{A}{C}, \quad G_{*}=\frac{B}{D} .
$$

They are single-valued on $M^{2}$. The geometric meaning of $G$ and $G_{*}$ is described in the Introduction. (See also [4].) By definition,

$$
d G=d\left(\frac{A}{C}\right)=\frac{d A C-A d C}{C^{2}}=\frac{B C-D A}{C^{2}} \omega=-\frac{\omega}{C^{2}} .
$$

Similarly,

$$
d G_{*}=d\left(\frac{B}{D}\right)=\frac{d B D-B d D}{D^{2}}=\frac{A D-B C}{D^{2}} \theta=\frac{\theta}{D^{2}} .
$$

On the other hand,

$$
G-G_{*}=\frac{A}{C}-\frac{B}{D}=\frac{A D-B C}{C D}=\frac{1}{C D} .
$$

We have the identity

$$
Q=\omega \theta=-(C D)^{2} d G d G_{*}=-\frac{d G d G_{*}}{\left(G-G_{*}\right)^{2}} .
$$

Now we set

$$
g(q):=\int_{p_{0}}^{q} \omega, \quad g_{*}(q):=\int_{p_{0}}^{q} \theta \quad\left(q \in M^{2}\right),
$$

where $p_{0}$ is a base point. Then $g$ and $g_{*}$ are holomorphic functions defined on $\widetilde{M}^{2}$. We remark that $(g, G)$ and $\left(g_{*}, G_{*}\right)$ satisfy the important relation

$$
S(g)-S(G)=2 Q, \quad S\left(g_{*}\right)-S\left(G_{*}\right)=2 Q,
$$

(see [4]), where $S(G)$ is the Schwarzian derivative

$$
S(G)=\left(\left(\frac{G^{\prime \prime}}{G^{\prime}}\right)^{\prime}-\frac{1}{2}\left(\frac{G^{\prime \prime}}{G^{\prime}}\right)^{2}\right) d z^{2} \quad\left({ }^{\prime}=\frac{d}{d z}\right)
$$


with respect to a local complex coordinate $z$ on $M^{2}$. Though the meromorphic 2-differentials $S(g)$ and $S(G)$ depend on complex coordinates, the difference $S(g)-S(G)$ does not.

Remark 1.1. Hyperbolic 3-space $H^{3}$ can be realized as a hyperboloid in Minkowski 4-space $\left(L^{4},\left(x^{0}, x^{1}, x^{2}, x^{3}\right)\right)$ :

$$
H^{3}=\left\{\left(x^{0}, x^{1}, x^{2}, x^{3}\right) \in L^{4} ; x^{0}>0,-\left(x^{0}\right)^{2}+\sum_{j=1}^{3}\left(x^{j}\right)^{2}=-1\right\} .
$$

Let $f: M^{2} \rightarrow H^{3}$ be a flat immersion and assume $M^{2}$ is connected. Then the universal cover $\widetilde{M}^{2}$ of $M^{2}$ is diffeomorphic to $\mathbf{R}^{2}$ and has a coordinate system $(x, y)$ defined on $\widetilde{M}^{2}$ such that the first fundamental form $d s^{2}$ can be written as

$$
d s^{2}=d x^{2}+d y^{2} .
$$

Then we have an orthonormal frame field

$$
e: \widetilde{M}^{2} \ni p \mapsto\left(f(p), f_{x}(p), f_{y}(p), \nu(p)\right) \in \mathrm{SO}(3,1),
$$

where $\nu(p) \in T_{p} H^{3}\left(\subset L^{4}\right)$ is the unit normal vector of the immersion $f$ at $p$. Now, we can identify $L^{4}$ with the set Herm(2) of 2 by 2 Hermitian matrices:

$$
L^{4} \ni\left(x^{0}, x^{1}, x^{2}, x^{3}\right) \longleftrightarrow\left(\begin{array}{cc}
x^{0}+x^{3} & x^{1}+i x^{2} \\
x^{1}-i x^{2} & x^{0}-x^{3}
\end{array}\right) \in \operatorname{Herm}(2) .
$$

Then the hyperbolic 3 -space $H^{3}$ can be rewritten as

$$
\begin{aligned}
H^{3} & =\{X \in \operatorname{Herm}(2) ; \operatorname{det}(X)=1, \operatorname{trace} X>0\} \\
& =\left\{a a^{*} ; a \in \mathrm{SL}(2, \mathbf{C})\right\},
\end{aligned}
$$

where $a^{*}={ }^{t} \bar{a}$. Setting

$$
v_{0}:=\left(\begin{array}{ll}
1 & 0 \\
0 & 1
\end{array}\right), \quad v_{1}:=\left(\begin{array}{ll}
0 & i \\
i & 0
\end{array}\right), \quad v_{2}:=\left(\begin{array}{rr}
0 & i \\
-i & 0
\end{array}\right), \quad v_{3}:=\left(\begin{array}{rr}
1 & 0 \\
0 & -1
\end{array}\right),
$$

there is a lift $E: \widetilde{M}^{2} \rightarrow \mathrm{SL}(2, \mathbf{C})$ of the orthonormal frame $e$ such that $e=$ $\pi \circ E$, where $\pi: \operatorname{SL}(2, \mathbf{C}) \rightarrow \mathrm{SO}(3,1)$ is the 2 -fold covering homomorphism, that is,

$$
f=E E^{*}, \quad f_{x}=E v_{1} E^{*}, \quad f_{y}=E v_{2} E^{*}, \quad \nu=E v_{3} E^{*} .
$$

Thus $E$ coincides with $E_{f}$. This implies that $E$ itself is holomorphic with respect to the complex structure induced from the second fundamental form. Multiplication $E \mapsto a E$ by a matrix $a=\left(a_{i j}\right) \in \mathrm{SL}(2, \mathbf{C})$ corresponds to an isometric change of the surface, $f \mapsto a f a^{*}$. This induces the change of hyperbolic Gauss maps, as follows:

$$
G \mapsto a \star G:=\frac{a_{11} G+a_{12}}{a_{21} G+a_{22}}, \quad G_{*} \mapsto a \star G_{*}=\frac{a_{11} G_{*}+a_{12}}{a_{21} G_{*}+a_{22}} .
$$


It is interesting to compare this with the case of surfaces of constant mean curvature 1 in $H^{3}$. In that case, there is a holomorphic immersion $F: \widetilde{M}^{2} \rightarrow \mathrm{SL}(2, \mathbf{C})$ such that $f=F F^{*}$, but it does not coincide with the lift $E: \widetilde{M}^{2} \rightarrow \mathrm{SL}(2, \mathbf{C})$ of an orthonormal frame. We must adjust $E$ by multiplying by a local $\mathrm{SU}(2)$-section $s: \widetilde{M}^{2} \rightarrow \mathrm{SU}(2)$ so that $F:=E s$ becomes holomorphic. (See Bryant [1].)

\section{Flat surfaces as (wave) fronts}

In this section, we define flat fronts as projections of Legendrian immersions into the unit cotangent bundle $T_{1}^{*} H^{3}$. Since $T_{1}^{*} H^{3}$ is isomorphic to the unit tangent bundle $T_{1} H^{3}$, we can make the identification

$$
T_{1}^{*} H^{3} \cong \mathcal{F}:=\left\{(x, v) \in L^{4} \times L^{4} ;-\langle x, x\rangle=\langle v, v\rangle=1,\langle x, v\rangle=0\right\},
$$

where $\langle$,$\rangle is the inner product of L^{4}$. The metric

$$
d \sigma_{0}^{2}:=\sum_{j=0}^{3}\left(d x^{j}\right)^{2}+\sum_{j=0}^{3}\left(d v^{j}\right)^{2} \quad\left(x=\left(x^{0}, x^{1}, x^{2}, x^{3}\right), v=\left(v^{0}, v^{1}, v^{2}, v^{3}\right)\right)
$$

on $\mathcal{F}$ induced from the product of Lorentzian metrics of $L^{4} \times L^{4}$ is positive definite, and is called the Sasakian metric. In fact, if we identify $\mathcal{F}$ with $T_{1} H^{3}$, it coincides with the metric on the unit tangent bundle defined by Sasaki $[\mathbf{9}, \mathbf{1 0}]$. The contact form of $\mathcal{F}$ is given by

$$
\eta:=\sum_{j=0}^{3} v^{j} d x^{j}
$$

Now, a Legendrian immersion $L$ of a 2-manifold $M^{2}$ into the unit cotangent bundle can be identified with an immersion

$$
L: M^{2} \longrightarrow \mathcal{F}
$$

such that $L^{*} \eta$ vanishes. We denote the two canonical projections by

$$
\pi_{F}: \mathcal{F} \ni(x, v) \longmapsto x \in H^{3}, \quad \pi_{F}^{\prime}: \mathcal{F} \ni(x, v) \longmapsto v \in L^{4} .
$$

A map $f: M^{2} \rightarrow H^{3}$ is called a front if there exists a Legendrian immersion $L_{f}: M^{2} \rightarrow \mathcal{F}$ such that

$$
\pi_{F} \circ L_{f}=f .
$$

$L_{f}$ is called the Legendrian lift of $f$.

By definition, any immersion $f: M^{2} \rightarrow H^{3}$ is a front if $M^{2}$ is orientable. In fact, $L_{f}$ is given by the pair $\left(f, \nu_{f}\right)$ consisting of $f$ and the unit normal vector $\nu_{f}$ of $f$.

For a given front $f: M^{2} \rightarrow H^{3}$, we can define a parallel front $f_{t}: M^{2} \rightarrow$ $H^{3}$ of distance $t$ by

$$
f_{t}:=(\cosh t) f+(\sinh t) \nu_{f}=\pi_{F} \circ L_{t},
$$


where

$$
L_{t}:=\left(f_{t}, \nu_{f_{t}}\right) \quad\left(\nu_{f_{t}}:=(\sinh t) f+(\cosh t) \nu_{f}\right)
$$

is a Legendrian immersion and

$$
\nu_{f}:=\pi_{F}^{\prime} \circ L_{f}: M^{2} \longrightarrow L^{4} .
$$

When $f$ is an immersion, this is nothing but the definition of a parallel surface. So we call $\nu_{f}$ the unit normal vector (field) of the front $f$.

For a given front $f: M^{2} \rightarrow H^{3}$,

$$
d s^{2}:=\langle d f, d f\rangle \quad \text { and } \quad d h^{2}:=-\left\langle d f, d \nu_{f}\right\rangle
$$

are called the first and the second fundamental forms, respectively.

Definition 2.1. A front $f: M^{2} \rightarrow H^{3}$ is called flat if, for each $p \in M^{2}$, there exists a real number $t \in \mathbf{R}$ such that the parallel front $f_{t}$ gives a flat immersion at $p$.

Remark 2.2. An equivalent definition of a flat front is that the Gaussian curvature of $f$ vanishes at all regular points. However, this definition is not suitable when all points of $f$ are degenerate, and such a case really occurs, since hyperbolic cylinders can collapse to a geodesic.

As shown in the following proposition, all parallel fronts $f_{t}(t \in \mathbf{R})$ of a flat front $f$ are also flat fronts.

Proposition 2.3. Let $f: M^{2} \rightarrow H^{3}$ be a flat front. Then the second fundamental form $d h^{2}$ is proportional to the pullback of the Sasakian metric $d \sigma^{2}=L_{f}^{*} d \sigma_{0}^{2}$. The parallel front $f_{t}$ of $f$ is also a flat front for all $t$. In particular, the Gaussian curvature of $f_{t}$ at the regular point vanishes.

Remark 2.4. As in [4], the lift $E_{f_{t}}$ of $f_{t}$ is given by

$$
E_{f_{t}}=E_{f}\left(\begin{array}{cc}
e^{t / 2} & 0 \\
0 & e^{-t / 2}
\end{array}\right) .
$$

Proof of Proposition 2.3. We fix a point $p \in M^{2}$. By definition, there is a parallel front $f_{t_{0}}: M^{2} \rightarrow H^{3}$ such that $f_{t_{0}}$ is regular at $p$ and the Gaussian curvature of $f_{t_{0}}$ vanishes around $p$. Without loss of generality, we may assume that $t_{0}=0$, that is, $f=f_{t_{0}}$.

First, we consider the case that the first and second fundamental forms are proportional. Then $f$ must be a horosphere and the statement of the theorem is obvious.

So we may assume that the second fundamental form is not proportional to the first. We can write the Legendrian lift $L_{f}$ as a pair $L_{f}=\left(f, \nu_{f}\right)$, where $\nu_{f}$ is the unit normal vector field of $f$. Then

$$
d \sigma^{2}=\langle d f, d f\rangle+\langle d \nu, d \nu\rangle .
$$


Now fix a local coordinate neighborhood $(U ; u, v)$ of $M^{2}$ and define three 2 by 2 matrices:

$$
\begin{aligned}
& M_{1}:=\left(\begin{array}{ll}
\left\langle f_{u}, f_{u}\right\rangle & \left\langle f_{u}, f_{v}\right\rangle \\
\left\langle f_{u}, f_{v}\right\rangle & \left\langle f_{v}, f_{v}\right\rangle
\end{array}\right), \\
& M_{2}:=-\left(\begin{array}{ll}
\left\langle f_{u}, \nu_{u}\right\rangle & \left\langle f_{u}, \nu_{v}\right\rangle \\
\left\langle f_{u}, \nu_{v}\right\rangle & \left\langle f_{v}, \nu_{v}\right\rangle
\end{array}\right), \\
& M_{3}:=\left(\begin{array}{ll}
\left\langle\nu_{u}, \nu_{u}\right\rangle & \left\langle\nu_{u}, \nu_{v}\right\rangle \\
\left\langle\nu_{u}, \nu_{v}\right\rangle & \left\langle\nu_{v}, \nu_{v}\right\rangle
\end{array}\right) .
\end{aligned}
$$

We set $A:=M_{1}^{-1} M_{2}$, which is the shape operator of $f$. The Gauss equation implies that

$$
\operatorname{det} A=1+K_{d s^{2}},
$$

where $K_{d s^{2}}$ is the Gaussian curvature of $d s^{2}$. On the other hand, by the definition of $M_{3}$, we have

$$
M_{3}=M_{2} A=M_{1} A^{2} .
$$

By the Cayley-Hamilton theorem, we have

$$
M_{3}=M_{1}\left(2(\operatorname{trace} A) A-\left(1+K_{d s^{2}}\right) I\right)=2(\operatorname{trace} A) M_{2}-K_{d s^{2}} M_{1},
$$

where $I$ is the identity matrix. Thus

$$
M_{1}+M_{3}-2(\operatorname{trace} A) M_{2}=-K_{d s^{2}} M_{1} .
$$

Since $M_{1}$ is not proportional to $M_{2}$, this implies that $M_{1}+M_{3}$ is proportional to $M_{2}$ if and only if $K_{d s^{2}}$ vanishes. So the second fundamental form $d h^{2}$ is proportional to $d \sigma^{2}$ when $f$ is flat. Now we shall show that $f_{t}$ is also flat. Indeed, $f_{t}$ and its unit normal vector $\nu_{t}$ have the expressions

$$
f_{t}=(\cosh t) f+(\sinh t) \nu, \quad \nu_{t}=(\sinh t) f+(\cosh t) \nu .
$$

The fundamental forms are

$$
\begin{aligned}
& d s_{t}^{2}=\left(\cosh ^{2} t\right) d s^{2}+2(\cosh t \sinh t) d h^{2}+\left(\sinh ^{2} t\right)\langle d \nu, d \nu\rangle, \\
& d h_{t}^{2}=(\cosh t \sinh t) d s^{2}+2\left(\cosh ^{2} t+\sinh ^{2} t\right) d h^{2}+(\cosh t \sinh t)\langle d \nu, d \nu\rangle, \\
& \left\langle d \nu_{t}, d \nu_{t}\right\rangle=\left(\sinh ^{2} t\right) d s^{2}+2(\cosh t \sinh t) d h^{2}+\left(\cosh ^{2} t\right)\langle d \nu, d \nu\rangle,
\end{aligned}
$$

where $\langle d \nu, d \nu\rangle$ is the third fundamental form of $f$. Since $d \sigma^{2}=d s^{2}+\langle d \nu, d \nu\rangle$, we have

$$
d h_{t}^{2}=(\cosh t \sinh t) d \sigma^{2}+2\left(\cosh ^{2} t+\sinh ^{2} t\right) d h^{2},
$$

and

$$
d \sigma_{t}^{2}:=d s_{t}^{2}+\left\langle d \nu_{t}, d \nu_{t}\right\rangle=\left(\cosh ^{2} t+\sinh ^{2} t\right) d \sigma^{2}+4 \cosh t \sinh t d h^{2} .
$$

Since $d h^{2}$ is proportional to $d \sigma^{2}, d h_{t}^{2}$ and $d \sigma_{t}^{2}$ are also proportional. Since $f$ is not a horosphere, $d s^{2}$ is not proportional to $d h^{2}$ and thus $f_{t}$ is flat for all $t \in \mathbf{R}$. 
From now on, we assume that $M^{2}$ is oriented. (If $M^{2}$ is not orientable, we can take the double cover.) Then there is a complex structure on $M^{2}$ such that $d \sigma^{2}$ is Hermitian. Since the second fundamental form is proportional to $d \sigma^{2}$, this complex structure of $M^{2}$ coincides with the one treated in Section 1, as long as $f$ is an immersion. So, we shall call this complex structure the canonical complex structure, and $M^{2}$ is always considered as a Riemann surface.

Proposition 2.5. Let $M^{2}$ be a Riemann surface and $E: \widetilde{M}^{2} \rightarrow \operatorname{SL}(2, \mathbf{C})$ a holomorphic Legendrian immersion defined on the universal cover $\widetilde{M}^{2}$ and such that $f=E E^{*}$ is single-valued on $M^{2}$. Then $f$ is a flat front. If

$$
E^{-1} d E=\left(\begin{array}{ll}
0 & \theta \\
\omega & 0
\end{array}\right)
$$

the first and the second fundamental forms are represented as

$$
\begin{aligned}
d s^{2} & =\omega \theta+\bar{\omega} \bar{\theta}+\left(|\omega|^{2}+|\theta|^{2}\right), \\
d h^{2} & =|\theta|^{2}-|\omega|^{2} .
\end{aligned}
$$

Conversely, any flat front is given as a projection of a holomorphic Legendrian immersion.

Proof. Let $E: \widetilde{M}^{2} \longrightarrow \mathrm{SL}(2, \mathbf{C})$ be a holomorphic Legendrian curve and let $(\omega, \theta)$ be as in (2.3). Then $E$ is an immersion if $|\omega|^{2}+|\theta|^{2}$ is positive definite. On the other hand, we have

$$
\begin{aligned}
d f & =d E E^{*}+E d E^{*}=E\left(E^{-1} d E+\left(E^{-1} d E\right)^{*}\right) E^{*} \\
& =E\left(\begin{array}{cc}
0 & \theta+\bar{\omega} \\
\omega+\bar{\theta} & 0
\end{array}\right) E^{*}, \\
d \nu & =d E v_{3} E^{*}+E v_{3} d E^{*}=E\left(E^{-1} d E v_{3}+v_{3}\left(E^{-1} d E\right)^{*}\right) E^{*} \\
& =E\left(\begin{array}{cc}
0 & -\theta+\bar{\omega} \\
\omega-\bar{\theta} & 0
\end{array}\right) E^{*} .
\end{aligned}
$$

In the identification as in (1.17), the canonical Lorentzian inner product is given by

$$
\langle X, Y\rangle:=-\frac{1}{2} \operatorname{trace}(X \tilde{Y}) \quad(X, Y \in \operatorname{Herm}(2)),
$$

where $\tilde{Y}$ is the cofactor matrix of $Y$. If $Y \in \operatorname{SL}(2, \mathbf{C})$, we have $\widetilde{Y}=Y^{-1}$. Then

$$
\begin{aligned}
d s^{2} & =\langle d f, d f\rangle=-\frac{1}{2} \operatorname{trace}\left\{\left(\begin{array}{cc}
0 & \theta+\bar{\omega} \\
\omega+\bar{\theta} & 0
\end{array}\right)\left(\begin{array}{cc}
0 & -\theta-\bar{\omega} \\
-\omega-\bar{\theta} & 0
\end{array}\right)\right\} \\
& =(\omega+\bar{\theta})(\bar{\omega}+\theta) .
\end{aligned}
$$


Similarly, since $d h^{2}=-\langle d f, d \nu\rangle$, we have (2.4). Thus, the pullback of the Sasakian metric by $(f, \nu)$ as in $(2.1)$ is represented as

$$
d \sigma^{2}=\langle d f, d f\rangle+\langle d \nu, d \nu\rangle=2\left(|\omega|^{2}+|\theta|^{2}\right) .
$$

Hence $L_{f}$ is an immersion if and only if $|\omega|^{2}+|\theta|^{2}$ is positive definite. This proves the assertion.

Remark 2.6. As seen in the proof of Proposition 2.5, the (1,1)-part of the first fundamental form

$$
d s_{1,1}^{2}=|\omega|^{2}+|\theta|^{2}
$$

is equal to one-half of $d \sigma^{2}$, the pullback of the Sasakian metric by $(f, \nu)$. Also, $d s_{1,1}^{2}$ is the pullback of the bi-invariant Hermitian metric of $\operatorname{SL}(2, \mathbf{C})$ by $E$.

A flat front $f: M^{2} \rightarrow H^{3}$ can be interpreted from two points of view. The first is the projection of a (real) Legendrian immersion $L_{f}: M^{2} \rightarrow T_{1}^{*} H^{3} \cong$ $\mathcal{F}$, and the second is the projection of a (holomorphic) Legendrian immersion $E_{f}: \widetilde{M}^{2} \rightarrow \mathrm{SL}(2, \mathbf{C})$. One can naturally expect that these two Legendrian immersions are related. In fact, $\operatorname{SL}(2, \mathbf{C})$ acts $\mathcal{F}$ transitively and we can write

$$
T_{1}^{*} H^{3} \cong T_{1} H^{3} \cong \mathcal{F} \cong \mathrm{SL}(2, \mathbf{C}) / \mathrm{U}(1) .
$$

We denote the canonical projection by

$$
p_{\mathrm{SL}}: \mathrm{SL}(2, \mathbf{C}) \longrightarrow \mathcal{F} \text {. }
$$

Proposition 2.7. The pullback of the contact form $\eta$ by $p_{\mathrm{SL}}$ is equal to the real part of the holomorphic contact form on $\mathrm{SL}(2, \mathbf{C})$, that is,

$$
p_{\mathrm{SL}}^{*}(\eta)=2 \operatorname{Re}\left(s_{22} d s_{11}-s_{12} d s_{21}\right)
$$

holds, where $\left(s_{i j}\right) \in \mathrm{SL}(2, \mathbf{C})$. In particular, the real Legendrian immersion $L_{f}$ can be interpreted as the projection of a holomorphic Legendrian immersion $E_{f}$.

Proof. Since

$$
x^{-1}=\left(s s^{*}\right)^{-1}=\left(s^{*}\right)^{-1} s^{-1}, \quad d v=s\left(s^{-1} d s v_{3}+v_{3}\left(s^{-1} d s\right)^{*}\right) s^{*},
$$

we have

$$
\begin{aligned}
\eta & =\langle x, d v\rangle=-\frac{1}{2} \operatorname{trace}\left(x^{-1} d v\right)=-\frac{1}{2} \operatorname{trace}\left(s^{-1} d s v_{3}+v_{3}\left(s^{-1} d s\right)^{*}\right) \\
& =-\operatorname{Re}\left(\operatorname{trace}\left(s^{-1} d s v_{3}\right)\right)=2 \operatorname{Re}\left(s_{22} d s_{11}-s_{21} d s_{12}\right),
\end{aligned}
$$

where we set $s=\left(s_{i j}\right) \in \mathrm{SL}(2, \mathbf{C})$. 
Remark 2.8. Since the holomorphic Legendrian lift $E_{f}$ of a flat front $f$ is not single-valued on $M^{2}$ in general, $E_{f}$ has the monodromy representation $\rho_{f}: \pi_{1}\left(M^{2}\right) \rightarrow \mathrm{SU}(2)$ such that $E_{f} \circ \tau=E_{f} \rho_{f}(\tau)$ for any deck transformation $\tau \in \pi_{1}(M)$. On the other hand, since $E_{f} \circ \tau$ is also Legendrian, the representation $\rho_{f}$ is reducible, that is, it reduces to the isotropy group $\mathrm{U}(1)$ of the action of $\operatorname{SL}(2, \mathbf{C})$ to $\mathcal{F}$.

We can define the hyperbolic Gauss maps of the flat front $f$ in the same way as for an immersion:

$$
G=\frac{A}{C}, \quad G_{*}=\frac{B}{D},
$$

where

$$
E_{f}=\left(\begin{array}{cc}
A & B \\
C & D
\end{array}\right)
$$

These are single-valued on $M^{2}$. Since $A D-B C=1, G(p)$ never coincides with $G_{*}(p)$, and we get the holomorphic map

$$
\mathcal{G}:=\left(G, G_{*}\right): M^{2} \longrightarrow S^{2} \times S^{2} \backslash\{\text { the diagonal set }\}=: \operatorname{Geod}\left(H^{3}\right),
$$

where $\operatorname{Geod}\left(H^{3}\right)$ is the set of oriented geodesics in $H^{3}$.

Theorem 2.9. Let $\widetilde{M}^{2}$ be the universal cover of a Riemann surface $M^{2}$ and let $E: \widetilde{M}^{2} \rightarrow \mathrm{SL}(2, \mathbf{C})$ be a holomorphic Legendrian curve such that $f=E E^{*}$ is single-valued on $M^{2}$. The following assertions are equivalent:

(1) $E$ is an immersion.

(2) $L_{f}$ is an immersion.

(3) The $(1,1)$-part of the first fundamental form

$$
d s_{1,1}^{2}=|\omega|^{2}+|\theta|^{2}
$$

is positive definite, where $\omega$ and $\theta$ are the off-diagonal components of $E^{-1} d E$.

(4) $\mathcal{G}:=\left(G, G_{*}\right): M^{2} \rightarrow \operatorname{Geod}\left(H^{3}\right)$ is an immersion.

Remark 2.10. A point where $E$ degenerates is called a branch point of $E$ (or of $f=E E^{*}$ ), and the projection of a holomorphic Legendrian curve is called a branched flat front. The conditions above imply that $f$ is free of branch points.

Proof of Theorem 2.9. The equivalence of the first three assertions follows from the proof of Proposition 2.5. So it is sufficient to prove that (3) and (4) are equivalent. By (1.11) and (1.12),

$$
d s_{1,1}^{2}=|\omega|^{2}+|\theta|^{2}=\left|C^{2} d G\right|^{2}+\left|D^{2} d G_{*}\right|^{2} .
$$

If both $C$ and $D$ are nonvanishing, the equivalence of (3) and (4) is obvious. If instead $C=0$, then $D \neq 0, \omega=0$,

$$
d s_{1,1}^{2}=|\theta|^{2}=\left|D^{2} d G_{*}\right|^{2},
$$


and $d s^{2}$ is positive definite if and only if $d G_{*} \neq 0$. Similarly, if $D=0$, we get $C \neq 0, \theta=0$,

$$
d s_{1,1}^{2}=|\omega|^{2}=\left|C^{2} d G\right|^{2},
$$

and $d s^{2}$ is positive definite if and only if $d G \neq 0$.

In [7], the authors gave a representation formula for Legendrian curves in $\operatorname{SL}(2, \mathbf{C})$ via the data $\left(G, G_{*}\right)$. We now reformulate it for the construction of flat fronts in $H^{3}$ :

Theorem 2.11. Let $G$ and $G_{*}$ be nonconstant meromorphic functions on a Riemann surface $M^{2}$ such that $G(p) \neq G_{*}(p)$ for all $p \in M^{2}$. Assume that:

(1) All poles of the 1 -form $\frac{d G}{G-G_{*}}$ are of order 1 .

(2) $\int_{\gamma} \frac{d G}{G-G_{*}}$ is purely imaginary for each loop $\gamma$ on $M^{2}$.

Set

$$
\xi(z):=c \exp \int_{z_{0}}^{z} \frac{d G}{G-G_{*}},
$$

where $z_{0} \in M^{2}$ is a base point and $c \in \mathbf{C} \backslash\{0\}$ is an arbitrary constant. Then

$$
E:=\left(\begin{array}{rr}
G / \xi & \xi G_{*} /\left(G-G_{*}\right) \\
1 / \xi & \xi /\left(G-G_{*}\right)
\end{array}\right)
$$

is a nonconstant meromorphic Legendrian curve defined on $\widetilde{M}^{2}$ in $\operatorname{SL}(2, \mathbf{C})$ whose hyperbolic Gauss maps are $G$ and $G_{*}$, and such that the projection $f=E E^{*}$ is single-valued on $M^{2}$. Moreover, $f$ is a front if and only if $G$ and $G_{*}$ have no common branch points. Conversely, any non-totally-umbilical flat fronts can be constructed in this manner.

Proof. Given a pair $\left(G, G_{*}\right)$ of nonconstant meromorphic functions, on a Riemann surface $M^{2}$ satisfying (1), the meromorphic map $E$ defined by (2.8) is a holomorphic Legendrian curve in $\operatorname{SL}(2, \mathbf{C})$, as a consequence of Theorem 3 of [7]. Then condition (2) implies that $f=E E^{*}$ is single-valued on $M^{2}$. Now, by Theorem 2.9 , the branched flat front $f$ is free of branch points if and only if the pair $\left(G, G_{*}\right)$ gives an immersion of $M^{2}$ into $S^{2} \times S^{2}$.

Since any flat front can be lifted to a holomorphic Legendrian curve defined on $\widetilde{M}^{2}$, Theorem 3 of [7] also implies that any non-totally-umbilical flat front can be constructed in this manner. (If one of the hyperbolic Gauss maps is constant, it is totally umbilic, i.e., locally a horosphere.)

\section{Flat fronts with complete ends}

We define completeness of fronts as follows: 
Definition 3.1. Let $M^{2}$ be a 2-manifold. A front $f: M^{2} \rightarrow H^{3}$ is called complete if there is a symmetric 2-tensor $T$ with compact support such that the sum

$$
T+d s^{2}
$$

is a complete Riemannian metric of $M^{2}$, where $d s^{2}$ is the first fundamental form of $f$.

Remark 3.2. Note that the parallel family of a complete front $f$ may contain an incomplete flat front. For example, the hyperbolic cylinder, that is, the surface equidistant from a geodesic (Example 4.1 in Section 4), contains a geodesic in its parallel family.

The following assertion is a simple consequence of Lemma 2 of [4]:

Lemma 3.3. Let $M^{2}$ be an oriented 2-manifold and $f: M^{2} \rightarrow H^{3}$ a complete flat front. There exists a compact Riemann surface $\bar{M}^{2}$ and finitely many points $p_{1}, \ldots, p_{n} \in \bar{M}^{2}$ such that $M^{2}$ (as a Riemann surface) is biholomorphic to $\bar{M}^{2} \backslash\left\{p_{1}, \ldots, p_{n}\right\}$. The Hopf differential $Q$ of $f$ can be extended meromorphically on $\bar{M}^{2}$.

These points $p_{1}, \ldots, p_{n}$ are called $e n d s$ of the front $f$.

Proof of Lemma 3.3. Since $f$ is complete, there exists a symmetric tensor $T$ supported in a compact subset of $M^{2}$ and such that

$$
d \bar{s}^{2}:=T+d s^{2}
$$

is complete, where $d s^{2}$ is the first fundamental form. Since the Gaussian curvature of $d s^{2}$ vanishes, the total absolute curvature of $d \bar{s}^{2}$ is finite. Then by Huber's theorem, there is a compact 2-manifold $\bar{M}^{2}$ and finite points $p_{1}, \ldots, p_{n} \in \bar{M}^{2}$ such that $M^{2}$ is diffeomorphic to $\bar{M}^{2} \backslash\left\{p_{1}, \ldots, p_{n}\right\}$. Now we take a sufficiently small neighborhood $U_{j}$ of an end $p_{j}$ such that $d \bar{s}^{2}=d s^{2}$ holds on $U_{j}$. If $|\omega|=|\theta|$ at a point $q \in U_{j}$, the first fundamental form $d s^{2}$ is degenerate at $q$ because of (1.5). Hence $|\omega| \neq|\theta|$ on $U_{j}$. If $|\omega|>|\theta|$, by (25) of [4], we have

$$
d s^{2}=\omega \theta+\bar{\omega} \bar{\theta}+|\omega|^{2}+|\theta|^{2} \leq 2|\omega||\theta|+|\omega|^{2}+|\theta|^{2}=(|\omega|+|\theta|)^{2} \leq 4|\omega|^{2} .
$$

Since $d s^{2}$ is complete at $p_{j}$, so is a metric $|\omega|^{2}$. Moreover, by the holomorphicity of $\omega$ with respect to the complex structure induced from the second fundamental form, the metric $|\omega|^{2}$ is a flat metric conformal to the complex structure of $M^{2}$ and complete at $p_{j}$. This proves the first assertion. (See also Lemma 1 of [4].) In the case of $|\omega|<|\theta|$, we reach the conclusion using the conformal metric $|\theta|^{2}$.

The meromorphicity of $Q$ is proved in Lemma 2 of [4].

As seen in the proof of Lemma 3.3, we have: 


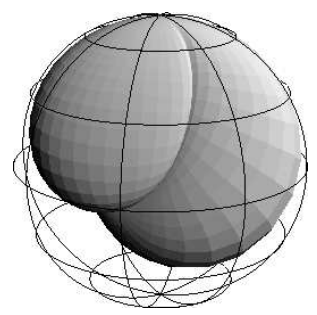

Figure 2. An incomplete flat front (Remark 3.5).

Corollary 3.4. If a flat front $f$ is complete, so is the $(1,1)$-part

$$
d s_{1,1}^{2}=|\omega|^{2}+|\theta|^{2}
$$

of the first fundamental form.

Remark 3.5. If a flat front is complete and is also a proper mapping, then its image is a closed subset of $H^{3}$. However, a proper flat front $f$ whose image is closed in $H^{3}$ may not be complete. (When $f$ has no singularity, it is complete by the Hopf-Rinow theorem.) In fact, we consider a flat front $f=E E^{*}: \mathbf{C} \rightarrow H^{3}$, where

$$
E:=\left(\begin{array}{rr}
z e^{-z} & (z-1) e^{z} \\
e^{-z} & e^{z}
\end{array}\right) .
$$

It can easily be checked that $f(z)$ tends to the north pole of the ideal boundary in the Poincaré ball as $z \rightarrow \infty$, which implies that $f$ is a proper mapping. But the first fundamental form vanishes on the imaginary axis, which appears as cuspidal edges. (See Figure 2. The criterion for singularities of flat fronts will appear in the forthcoming paper $[6]$.)

It is a classical fact that there are no compact flat surfaces in $H^{3}$. We can also prove the nonexistence of compact flat fronts.

Proposition 3.6. There are no compact flat fronts without boundary.

Proof. Let $f: M^{2} \rightarrow H^{3}$ be a compact flat front, and take a holomorphic Legendrian lift $E$ of $f$. Recall that $f$ and $E$ are matrix-valued. The trace of $f$ satisfies

$$
(\operatorname{trace} f)_{z \bar{z}}=\operatorname{trace}\left(f_{z \bar{z}}\right)=\operatorname{trace}\left\{E_{z}\left(E_{z}\right)^{*}\right\} \geq 0,
$$

where $z$ is a complex coordinate of $M^{2}$. Hence the function trace $f: M^{2} \rightarrow \mathbf{R}$ is subharmonic, and must be constant, since $M^{2}$ is compact. By an isometry in $H^{3}$, we may assume that $f\left(z_{0}\right)=I$, where $I$ is the $2 \times 2$ identity matrix. Then trace $f$ is identically 2 . At the same time, $\operatorname{det} f$ is identically 1 so the eigenvalues $\lambda_{1}, \lambda_{2}$ of $f$ satisfy

$$
\lambda_{1}+\lambda_{2}=2, \quad \lambda_{1} \lambda_{2}=1 .
$$


Hence $\lambda_{1}=\lambda_{2}=1$. Since $f$ is Hermitian, this implies that $f(z)$ is equal to the identity matrix, a contradiction.

Gálvez, Martínez and Milán investigated complete ends of flat surfaces deeply. The following fact is proved in [4]:

Lemma 3.7 (Theorem 4 of [4]). Let $p$ be an end of a complete flat front. The following three conditions are equivalent:

(1) The Hopf differential $Q$ has at most a pole of order 2 at $p$.

(2) The positive hyperbolic Gauss map $G$ has at most a pole at $p$.

(3) The negative hyperbolic Gauss map $G_{*}$ has at most a pole at $p$.

Remark 3.8. The hyperbolic Gauss maps and the Hopf differential of the flat front $f=E E^{*}$ as in (3.1) are

$$
G=z, \quad G_{*}=z-1, \quad Q=d z^{2} .
$$

This means that meromorphicity of $G$ and $G_{*}$ does not imply that $Q$ has at most poles of order 2 without assuming the completeness of ends. In fact, $Q$ has pole of order 4 at $z=\infty$.

If an end of a flat front satisfies one of the three conditions above, it is called a regular end. An end that is not regular is called an irregular end. An end $p$ is said to be embedded if there is a neighborhood $U$ of $p \in \bar{M}^{2}$ such that the restriction of the front to $U \backslash\{p\}$ is an embedding.

Lemma 3.9 (Theorem 5 of [4]). Let $p$ be a regular end of a complete flat front. Suppose that $|\theta|<|\omega|$ at $p$. Then $p$ is embedded if and only if it is not a branch point of the positive hyperbolic Gauss map $G$.

Lemma 3.10. The two hyperbolic Gauss maps take the same value at a regular end of a complete flat front. That is, $G(p)=G_{*}(p)$ if $p$ is a regular end.

Proof. Assume that $G(p) \neq G_{*}(p)$ for a regular end $p$. By Lemma 3.7, $G(z)$ and $G_{*}(z)$ are both meromorphic at $p$. In particular, the function $\xi(z)$ defined in Theorem 2.11 is holomorphic. Then so is $E$, contradicting the completeness of the first fundamental form of the front at $p$. Thus $G(p)=G_{*}(p)$.

Let $f: \bar{M}^{2} \backslash\left\{p_{1}, \ldots, p_{n}\right\} \rightarrow H^{3}$ be a complete flat front and $\omega, \theta$ its first and second canonical forms. Suppose all ends $p_{1}, \ldots, p_{n}$ are regular. By Lemma 2 of [4], there exist real numbers $\mu_{j}$ and $\mu_{j}^{*}(j=1, \ldots, n)$ such that

$$
\begin{array}{ll}
\omega(z)=\left(z-p_{j}\right)^{\mu_{j}} \omega_{0}(z) & \left(\omega_{0}\left(p_{j}\right) \neq 0\right), \\
\theta(z) & =\left(z-p_{j}\right)^{\mu_{j}^{*}} \theta_{0}(z) \quad\left(\theta_{0}\left(p_{j}\right) \neq 0\right),
\end{array}
$$

where $(U ; z)$ is a complex coordinate around $p_{j}$ and $\omega_{0}(z)$ and $\theta_{0}(z)$ are holomorphic 1-forms defined on $U$. The real numbers $\mu_{j}$ and $\mu_{j}^{*}$ do not depend 
on the choice of the coordinate $z$ and equal the order of the pseudometrics $|\omega|^{2}$ and $|\theta|^{2}$, respectively:

$$
\begin{aligned}
& |\omega|^{2}=\left|z-p_{j}\right|^{2 \mu_{j}}\left(a_{j}+o(1)\right)|d z|^{2}, \\
& |\theta|^{2}=\left|z-p_{j}\right|^{2 \mu_{j}^{*}}\left(a_{j}^{*}+o(1)\right)|d z|^{2},
\end{aligned}
$$

where $a_{j}$ and $a_{j}^{*}$ are positive real numbers and $o(1)$ denotes higher order terms. By (1.14), we have

$$
\mu_{j}+\mu_{j}^{*}=\operatorname{ord}_{p_{j}} Q,
$$

where $\operatorname{ord}_{p_{j}} Q$ is the order of the Hopf differential $Q$ at $p_{j}$. Suppose that the Laurent expansion of $Q$ at $z=p_{j}$ is

$$
Q=\frac{1}{\left(z-p_{j}\right)^{2}}\left(q_{-2}\left(p_{j}\right)+o(1)\right) d z^{2},
$$

where $o(1)$ is a function satisfying $\lim _{z \rightarrow p_{j}} o(1)=0$. The following lemma is a direct consequence of the formula (1.16):

Lemma 3.11. The identity

$$
4 q_{-2}\left(p_{j}\right)=m_{j}\left(m_{j}+2\right)-\mu_{j}\left(\mu_{j}+2\right)=m_{j}^{*}\left(m_{j}^{*}+2\right)-\mu_{j}^{*}\left(\mu_{j}^{*}+2\right)
$$

holds, where $\mu_{j}$ and $\mu_{j}^{*}$ are the orders of the pseudometrics $|\omega|^{2}$ and $|\theta|^{2}$, respectively, and $m_{j}$ and $m_{j}^{*}$ are the branching orders of $G$ and $G_{*}$ at $p_{j}$. (For instance, $m_{j}=1$ implies $p_{j}$ is a double point of $G$.)

Proposition 3.12. A regular end $p_{j}$ of a complete flat front is embedded if and only if either $G$ or $G_{*}$ does not branch at $p_{j}$.

Proof. First, we assume the end $p_{j}$ is embedded. As pointed out in a previous paper [7], the holomorphic Legendrian lift $E_{f}$ enjoys the following duality. We set

$$
\hat{E}_{f}:=E_{f}\left(\begin{array}{cc}
0 & i \\
i & 0
\end{array}\right)
$$

Then $\hat{E}_{f}$ is also a holomorphic Legendrian immersion such that $f=\hat{E}_{f} \hat{E}_{f}^{*}$, and the roles of $(G, \omega)$ and $\left(G_{*}, \theta\right)$ are interchanged. Then, replacing $E_{f}$ by $\hat{E}_{f}$ if necessary, we may assume $|\theta|<|\omega|$ near $p_{j}$. By Lemma 3.9, $G$ does not branch at $p_{j}$.

Conversely, we assume either $G$ or $G_{*}$ does not branch at $p_{j}$. Replacing $E_{f}$ by $\hat{E}_{f}$ if necessary, we may assume $G$ does not branch at $p_{j}$, that is, $m_{j}=0$. If $|\theta|<|\omega|$ near $p_{j}$, the assertion follows from Lemma 3.9. So we may assume $|\theta|>|\omega|$ near $p_{j}$, and then we have $\mu_{j} \geq \mu_{j}^{*}$. By Lemma 3.11,

$$
\left(m_{j}-m_{j}^{*}\right)\left(m_{j}+m_{j}^{*}+2\right)=\left(\mu_{j}-\mu_{j}^{*}\right)\left(\mu_{j}+\mu_{j}^{*}+2\right) .
$$

By (3.3),

$$
\mu_{j}+\mu_{j}^{*}+2=\operatorname{ord}_{p_{j}} Q+2 .
$$


Since $p_{j}$ is regular, $\operatorname{ord}_{p_{j}} Q \geq-2$. Thus $0=m_{j} \geq m_{j}^{*}(\geq 0)$, and so neither $G$ nor $G_{*}$ branch at $p$. Replacing $E_{f}$ by $\hat{E}_{f}$ if necessary, we may assume $|\theta|<|\omega|$ and get the embeddedness of $p$ directly from Lemma 3.11.

Now we shall prove an assertion stated in the Introduction:

Theorem 3.13. Let $f: \bar{M}^{2} \backslash\left\{p_{1}, \ldots, p_{n}\right\} \rightarrow H^{3}$ be a complete flat front whose ends are all regular. Then

$$
\operatorname{deg} G+\operatorname{deg} G_{*} \geq n,
$$

and equality holds if and only if all ends are embedded.

To prove the theorem, we shall prepare two lemmas.

Lemma 3.14. Let $g$ and $h$ be meromorphic functions on a compact Riemann surface $\bar{M}^{2}$. Suppose that $g$ and $h$ have no common poles. Then

$$
\operatorname{deg}(a g+b h)=\operatorname{deg} g+\operatorname{deg} h,
$$

where $a, b \in \mathbf{C}$ are nonzero constants.

Proof. Since $g, h$ are meromorphic, their degrees equal the number of their poles, counting multiplicities. If $P(g)$ is the divisor of poles of $g$ (so that $P(g)=s_{1} q_{1}+\cdots+s_{n} q_{n}$, with $q_{1}, \ldots, q_{n}$ the poles of $g$ and $s_{1}, \ldots, s_{n}$ their multiplicities), the degree of $g$ is the sum of the coefficients of $P(g)$, and likewise for $h$. But $P(a g+b h)=P(g)+P(h)$ unless $a b=0$. Thus

$$
\operatorname{deg} g+\operatorname{deg} h=\operatorname{deg}(a g+b h) .
$$

Lemma 3.15. Let $f: \bar{M}^{2} \backslash\left\{p_{1}, \ldots, p_{n}\right\} \rightarrow H^{3}$ be a complete flat front. Suppose that $p=p_{j}$ is a regular end. Then $p$ is an embedded end if and only if the difference

$$
h:=G-G_{*}
$$

of the two hyperbolic Gauss maps does not branch at $p$.

Proof. If $p$ is not a branch point of $h$, then either $G$ or $G_{*}$ does not branch at $p$. Then embeddedness of the end $p=p_{j}$ follows from Proposition 3.12. Conversely, suppose now that an end $p$ is embedded. We take a complex coordinate $z$ around $p$ such that $z(p)=0$. Then, by an isometry of $H^{3}$, we may assume $G(0)=G_{*}(0)=0$ because of Lemma 3.10 and (1.19). It follows from Proposition 3.12 that $G$ and $G_{*}$ are expanded as

$$
G(z)=a z+o(z) \quad \text { and } \quad G_{*}(z)=a_{*} z+o(z),
$$

where $a$ and $a_{*}$ are complex numbers such that $a \neq 0$ or $a_{*} \neq 0$, and $o(z)$ denotes a higher order term. Thus by (1.14), the Hopf differential $Q$ has the expansion

$$
Q=-\frac{a a_{*}+o(1)}{\left(\left(a-a_{*}\right) z+o(z)\right)^{2}} d z^{2} .
$$


By Lemma 3.7, we have $a a_{*}=0$ or $a-a_{*} \neq 0$. If $a-a_{*} \neq 0$, it follows that $h$ does not branch at 0 . If $a a_{*}=0$, one of $G$ and $G_{*}$ branches at 0 and the other does not. Then $h=G-G_{*}$ does not branch at 0 .

Proof of Theorem 3.13. Taking an isometry if necessary, we may assume that all ends $p_{1}, \ldots, p_{n}$ are not poles of both hyperbolic Gauss maps $G$ and $G_{*}$. The ends of the front are equal to the zeros of $h:=G-G_{*}$, so $G$ and $G_{*}$ have no common poles. The zero divisor $Z(h)$ of the meromorphic function $h$ is of the form

$$
Z(h)=\sum_{j=1}^{n} m_{j} p_{j},
$$

where $m_{1}, \ldots, m_{n}$ are positive integers. Then by Lemma 3.14

$$
\operatorname{deg} G+\operatorname{deg} G_{*}=\operatorname{deg} h=\sum_{j=1}^{n} m_{j} \geq n,
$$

which proves the inequality. Moreover, the equality holds if and only if

$$
m_{1}=\cdots=m_{n}=1 .
$$

By Lemma 3.15, this is the case if and only if all ends are embedded.

Remark 3.16. As seen in the proof, the inequality in Theorem 3.13 holds even if $f$ has branch points (see Remark 2.10), that is, common branch points of $G$ and $G_{*}$ on $M^{2}$. However, the category of branched flat front seems too wide for the study of flat surfaces. In fact, branched covers of flat fronts are all branched flat fronts whose images are the same as the original fronts.

Remark 3.17. Let $\bar{M}^{2}$ be a compact Riemann surface with positive genus. Since there are no meromorphic functions on $\bar{M}^{2}$ of degree 1, a complete flat front defined on $\bar{M}^{2}$ minus a finite number of points must have at least 4 ends. Is there a flat front with positive genus and exactly 4 embedded ends? There does exist a genus-1 flat front with 5 ends (Example 4.6), but it is still unknown whether there one with 4 ends. (One can construct a genus-1 branched flat front with 4 embedded ends, but the image of such a front is a double cover of a genus-0 flat front.)

As seen in Remark 3.16, the inequality of Theorem 3.13 is valid for branched flat fronts. On the other hand, we show an inequality which reflects properties of fronts. First some terminology:

Definition 3.18. Let $p$ be a regular end of a flat front and let $(\omega, \theta)$ be as in (2.3). The end $p$ is called cylindrical if

$$
\operatorname{ord}_{p}|\omega|^{2}=\operatorname{ord}_{p}|\theta|^{2}=-1,
$$


where $\operatorname{ord}_{p}|\omega|^{2}$ and $\operatorname{ord}_{p}|\theta|^{2}$ are the orders of the pseudometrics $|\omega|^{2}$ and $|\theta|^{2}$, as in (3.2), respectively.

The ends of a hyperbolic cylinder, a surface equidistant from a geodesic, are cylindrical. (See Example 4.1, and also [4, p. 427] or [7, Example 4.1].)

Lemma 3.19 (Theorem 6 of [4]). A cylindrical end is asymptotic to a finite cover of a hyperbolic cylinder.

Lemma 3.20. A regular end $p$ of a complete flat front is cylindrical if and only if $\operatorname{ord}_{p}|\omega|^{2}=-1$ or $\operatorname{ord}_{p}|\theta|^{2}=-1$.

Proof. Assume $\operatorname{ord}_{p}|\omega|=-1$. Then $\omega$ is written as $\omega=(z-p)^{-1} \omega_{0}(z)$, where $\omega_{0}$ is a holomorphic 1-form such that $\omega_{0}(p) \neq 0$ and $z$ is a complex coordinate around $p$. After an isometry of $H^{3}$ if necessary, we may assume $G(p)=0$ because of (1.19). Then $G$ is written as $(z-p)^{m} G_{0}(z)$, where $m \geq 1$ is an integer and $G_{0}(z)$ is a holomorphic function such that $G_{0}(p) \neq 0$. From (1.16) we conclude that the order of the Hopf differential $Q$ at $z=p$ is -2 . Hence by (1.9), $\operatorname{ord}_{p}|\theta|^{2}=-1$. Similarly, if $\operatorname{ord}_{p}|\theta|^{2}=-1$, we have $\operatorname{ord}_{p}|\omega|^{2}=-1$.

Let $f: \bar{M}^{2} \backslash\left\{p_{1}, \ldots, p_{n}\right\} \rightarrow H^{3}$ be a complete flat front and let $(\omega, \theta)$ be as in (2.3). By (2.5), the pullback of the Sasakian metric of $T_{1}^{*} H^{3}$ by the Legendrian lift of $f$ is

$$
d \sigma^{2}=2\left(|\omega|^{2}+|\theta|^{2}\right) .
$$

Since $\omega$ and $\theta$ are holomorphic 1-forms, the Gaussian curvature $K_{d \sigma^{2}}$ of $d \sigma^{2}$ is nonpositive. Moreover, $d \sigma^{2}$ is complete because of Corollary 3.4. Thus it satisfies the Cohn-Vossen inequality (0.1) in the Introduction.

Proposition 3.21. Equality holds in (0.1) if and only if all ends are regular and cylindrical.

Proof. In fact,

$$
\begin{aligned}
\frac{1}{2 \pi} \int_{M^{2}}\left(-K_{d \sigma^{2}}\right) d A_{d \sigma^{2}} & =-\chi\left(\bar{M}^{2}\right)+\sum_{j=1}^{n} \operatorname{ord}_{p_{j}} d \sigma^{2} \\
& =-\chi\left(M^{2}\right)+\sum_{j=1}^{n}\left(\operatorname{ord}_{p_{j}} d \sigma^{2}+1\right) .
\end{aligned}
$$

(See [11] or Corollary 1 of [3].) In our case $\operatorname{ord}_{p_{j}} d \sigma^{2} \leq-1$, since $d \sigma^{2}$ is complete. Hence equality holds if and only if $\operatorname{ord}_{p_{j}} d \sigma^{2}=-1$ for all $j=1, \ldots, n$. But by $(3.4)$,

$$
\operatorname{ord}_{p_{j}} d \sigma^{2}=\min \left\{\operatorname{ord}_{p_{j}}|\omega|^{2}, \operatorname{ord}_{p_{j}}|\theta|^{2}\right\} .
$$

Hence if $\operatorname{ord}_{p_{j}} d \sigma^{2}=-1$, the end $p_{j}$ is regular because of (1.9) and Lemma 3.7, and then $p_{j}$ is cylindrical because of Lemma 3.20. 
Note that the left-hand side of (0.1) may not be an integer.

\section{Examples and a classification}

In this section, we investigate complete flat fronts all of whose ends are regular and embedded. We shall classify them when the number of ends is less than or equal to 3 . We begin by reviewing known examples and their hyperbolic Gauss maps.

Example 4.1 (flat fronts of revolution). Let $\bar{M}^{2}$ denote the Riemann sphere $S^{2}=\mathbf{C} \cup\{\infty\}$ and consider a pair $\left(G, G_{*}\right)$ of meromorphic functions on $\bar{M}^{2}$ defined by $G(z)=z$ and $G_{*}(z)=\alpha z$, for some constant $\alpha \in \mathbf{R} \backslash\{1\}$. Define $M^{2}$ by

$$
M^{2}:= \begin{cases}\bar{M}^{2} \backslash\{0\} & \text { if } \alpha=0 \\ \bar{M}^{2} \backslash\{0, \infty\} & \text { otherwise. }\end{cases}
$$

One can easily check that $M^{2}$ and $\left(G, G_{*}\right)$ satisfy conditions (1) and (2) of Theorem 2.11. Indeed, these data give a Legendrian immersion

$$
E=\left(\begin{array}{cc}
\frac{z^{-\alpha /(1-\alpha)}}{c} & \frac{c \alpha z^{1 /(1-\alpha)}}{1-\alpha} \\
\frac{z^{-1 /(1-\alpha)}}{c} & \frac{c z^{\alpha /(1-\alpha)}}{1-\alpha}
\end{array}\right) \text { for some constant } c
$$

and a resulting flat front $f:=E E^{*}: M^{2} \rightarrow H^{3}$.

This flat front $f$ is a horosphere if $\alpha=0$ or a hyperbolic cylinder if $\alpha=-1$. We shall call $f$ an hourglass if $\alpha(\neq-1)<0$, or a snowman if $\alpha>0$. The first and second canonical forms and the Hopf differential are

$$
\omega=-\frac{1}{c^{2}} z^{-2 /(1-\alpha)} d z, \quad \theta=\frac{c^{2} \alpha}{(1-\alpha)^{2}} z^{2 \alpha /(1-\alpha)} d z, \quad Q=-\frac{\alpha}{(1-\alpha)^{2}} z^{2} d z^{2} .
$$

The total curvature of the pullback of the Sasakian metric is calculated as

$$
\frac{1}{2 \pi} \int_{M^{2}}\left(-K_{d \sigma^{2}}\right) d A_{d \sigma^{2}}=2\left|\frac{1+\alpha}{1-\alpha}\right| \text {. }
$$

Horospheres can be characterized by the hyperbolic Gauss maps.

Proposition 4.2. Let $f: M^{2} \rightarrow H^{3}$ be a complete flat front. Assume that one of the hyperbolic Gauss maps $G, G_{*}$ is constant. Then $f$ is a horosphere.

Proof. It suffices to prove the case of $G_{*}$ constant. In this case, $G$ must be nonbranched, because $\mathcal{G}=\left(G, G_{*}\right)$ is an immersion. On the other hand, $Q$ is identically zero. It follows from Lemma 3.7 that $G$ has at most poles, that is, $G$ is a meromorphic function on a compact Riemann surface $\bar{M}^{2}$. This implies that $G$ gives a biholomorphism $\bar{M}^{2} \cong S^{2}$. Therefore we may assume 


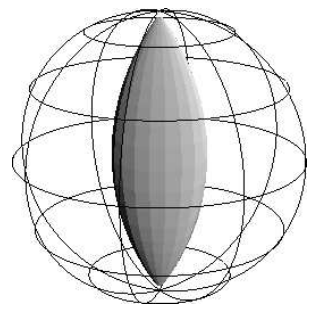

$\alpha=-1$

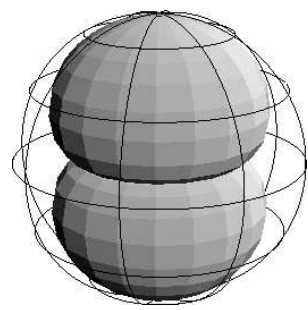

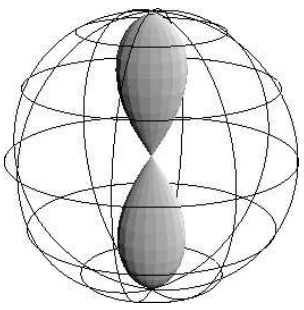

$\alpha<0(\alpha \neq-1)$

$\alpha>0$

Figure 3. Flat fronts of revolution.

$G(z)=z$ on $\bar{M}^{2}\left(\cong S^{2} \cong \mathbf{C} \cup\{\infty\}\right)$. Then it follows from Example 4.1 that $f$ is a horosphere.

Lemma 4.3. Let $f: M^{2}=\bar{M}^{2} \backslash\left\{p_{1}, \ldots, p_{n}\right\} \rightarrow H^{3}$ be a complete flat front with embedded regular ends $p_{1}, \ldots, p_{n}$. If $n \leq 3$, then $\bar{M}^{2}$ is biholomorphic to the Riemann sphere.

Proof. By Proposition 4.2, it suffices to prove this when both $G$ and $G_{*}$ are nonconstant, i.e., $\operatorname{deg} G \geq 1$ and $\operatorname{deg} G_{*} \geq 1$. Since all ends are regular and embedded, $\operatorname{deg} G+\operatorname{deg} G_{*}=n \leq 3$. Therefore $\operatorname{deg} G=1$ or $\operatorname{deg} G_{*}=1$. Thus $G$ or $G_{*}$ is a biholomorphism to the Riemann sphere.

Let us investigate complete flat fronts $f: \bar{M}^{2} \backslash\left\{p_{1}, p_{2}\right\} \rightarrow H^{3}$ with 2 embedded regular ends $p_{1}, p_{2}$. As stated in Lemma $4.3, \bar{M}^{2}=S^{2} \cong \mathbf{C} \cup\{\infty\}$. Without loss of generality, we may assume that the images of the 2 ends are $0, \infty \in S^{2}\left(=\partial H^{3}\right)$ :

$$
G\left(p_{1}\right)=G_{*}\left(p_{1}\right)=0, \quad G\left(p_{2}\right)=G_{*}\left(p_{2}\right)=\infty .
$$

Since the ends are embedded, $G$ and $G_{*}$ have degree 1 . We identify $\bar{M}^{2}$ with $S^{2}$ via $G$, that is, $G(z)=z$. Then the coordinates of $p_{1}, p_{2}$ are $z=0, \infty$, respectively. We can also set $G_{*}(z)=(a z+b) /(c z+d)$. It follows from (4.3) that $b=c=0$. Therefore $G_{*}(z)=\alpha z$ for some nonzero constant $\alpha$. Moreover, conditions (1) and (2) of Theorem 2.11 imply $\alpha \in \mathbf{R} \backslash\{0,1\}$.

To summarize, $f$ is congruent to a flat front of $\left(G, G_{*}\right)=(z, \alpha z)$ for some $\alpha \in \mathbf{R} \backslash\{0,1\}$. Hence, it is a flat front of revolution (see Example 4.1). 
Next we investigate complete flat fronts $f: \bar{M}^{2} \backslash\left\{p_{1}, p_{2}, p_{3}\right\} \rightarrow H^{3}$, called trinoids, with 3 embedded regular ends $p_{1}, p_{2}, p_{3}$. We may assume $\bar{M}^{2}=$ $S^{2} \cong \mathbf{C} \cup\{\infty\}$ by Lemma 4.3 and $\operatorname{deg} G=1, \operatorname{deg} G_{*}=2$. As in the case of 2 -end fronts above, we may assume that $G(z)=z$ and that

$$
G_{*}(0)=0, \quad G_{*}(1)=1, \quad G_{*}(\infty)=\infty
$$

are the images of the ends. Since $G_{*}$ is a meromorphic function on $\bar{M}^{2}=S^{2}$ of degree 2 , it is a quotient of polynomials of degree $\leq 2$. Indeed, one can check from (4.4) that $G_{*}$ has the form

$$
G_{*}(z)=\frac{z(\alpha z+\beta)}{\gamma z+1},
$$

where $\alpha, \beta, \gamma \in \mathbf{C}$ satisfy

$$
\alpha+\beta=\gamma+1, \quad \alpha \neq 0, \quad \alpha-\beta \gamma \neq 0 .
$$

The conditions (4.6) can be rewritten as

$$
\beta=-\alpha+\gamma+1, \quad \alpha(\alpha-\gamma)(\gamma+1) \neq 0 .
$$

By straightforward computation, we see that

$$
\frac{d G}{G-G_{*}}=\left(\frac{1}{\gamma-\alpha}\right) \frac{\gamma z+1}{z(z-1)} d z
$$

which has poles only at $z=0,1, \infty$. All of them are simple poles, with residues $-1 /(\gamma-\alpha),(\gamma+1) /(\gamma-\alpha),-\gamma /(\gamma-\alpha)$, respectively. These residues must be real, because of condition (2) of Theorem 2.11. Hence $\alpha, \gamma \in \mathbf{R}(\beta \in \mathbf{R})$. It follows from Theorem 2.11 and (1.11) that

$$
\begin{aligned}
& \xi\left(=c \exp \int \frac{d G}{G-G_{*}}\right)=c z^{1 /(\alpha-\gamma)}(z-1)^{(\gamma+1) /(\gamma-\alpha)}, \\
& \omega\left(=-\xi^{-2} d G\right)=-c^{-2} z^{2 /(\gamma-\alpha)}(z-1)^{(2 \gamma+2) /(\alpha-\gamma)} d z .
\end{aligned}
$$

The Hopf differential $Q$ is computed as

$$
Q\left(=-\frac{d G d G_{*}}{\left(G-G_{*}\right)^{2}}\right)=-\frac{1}{(\gamma-\alpha)^{2}} \frac{\alpha \gamma z^{2}+2 \alpha z+\beta}{z^{2}(z-1)^{2}} d z^{2} .
$$

Thus $Q$ has poles only at $z=0,1, \infty$ with orders at most 2 . Indeed,

$\left(\operatorname{ord}_{0} Q, \operatorname{ord}_{1} Q, \operatorname{ord}_{\infty} Q\right)= \begin{cases}(-1,-2,-2) & \text { if } \alpha=\gamma+1(\Longleftrightarrow \beta=0), \\ (-2,-1,-2) & \text { if } \alpha=-1(\Longleftrightarrow \alpha \gamma+2 \alpha+\beta=0), \\ (-2,-2,-1) & \text { if } \gamma=0(\Longleftrightarrow \alpha \gamma=0), \\ (-2,-2,-2) & \text { otherwise. }\end{cases}$

To summarize, we have obtained the following classification theorem:

Theorem 4.4. Let $f: M^{2} \rightarrow H^{3}$ be a complete flat front of which all ends are regular and embedded. If $f$ has at most 3 ends, it is congruent to one of the following: 
(i) a horosphere if it has a single end,

(ii) a hyperbolic cylinder, an hourglass, or a snowman if it has 2 ends,

(iii) a trinoid with

$$
\left(G, G_{*}\right)=\left(z, \frac{z(\alpha z+\beta)}{\gamma z+1}\right),
$$

where $\alpha, \beta, \gamma$ are real constants satisfying (4.7), if it has 3 ends.

For arbitrary distinct points $p_{1}, \ldots, p_{n} \in \partial H^{3}=\mathbf{C} \cup\{\infty\}$, we can construct a flat front of genus zero with embedded regular ends $p_{1}, \ldots, p_{n}$ as follows:

Example 4.5. Let $p_{1}, \ldots, p_{n}$ be arbitrary distinct points in $\partial H^{3}=\mathbf{C} \cup$ $\{\infty\}$. Without loss of generality, we may assume that $p_{n}=\infty$. Choose nonzero real numbers $a_{1}, \ldots, a_{n-1}$ such that $a_{1}+\cdots+a_{n-1} \neq 0,1$. Set

$$
\begin{aligned}
M^{2} & =\mathbf{C} \backslash\left\{p_{1}, \ldots, p_{n-1}\right\}, \\
G & =z \\
G_{*} & =\left(z \sum_{k=1}^{n-1}\left(a_{k} \prod_{j \neq k}\left(z-p_{j}\right)\right)-\prod_{j=1}^{n-1}\left(z-p_{j}\right)\right) / \sum_{k=1}^{n-1}\left(a_{k} \prod_{j \neq k}\left(z-p_{j}\right)\right) .
\end{aligned}
$$

Then

$$
\frac{d G}{G-G_{*}}=\left(\frac{a_{1}}{z-p_{1}}+\frac{a_{1}}{z-p_{1}}+\cdots+\frac{a_{n-1}}{z-p_{n-1}}\right) d z .
$$

It follows that $M^{2}$ and $\left(G, G_{*}\right)$ satisfy the conditions of Theorem 2.11 . Therefore these data yield a flat front. A straightforward computation shows that

$$
\begin{aligned}
& \xi=c \prod_{j=1}^{n-1}\left(z-p_{j}\right)^{a_{j}}, \\
& \omega\left(=-d G / \xi^{2}\right)=-c^{-2}\left(\prod_{j=1}^{n-1}\left(z-p_{j}\right)^{-2 a_{j}}\right) d z \\
& Q=\left(\sum_{j=1}^{n-1} \frac{a_{j}}{\left(z-p_{j}\right)^{2}}-\left(\sum_{j=1}^{n-1} \frac{a_{j}}{z-p_{j}}\right)^{2}\right) d z^{2} .
\end{aligned}
$$

It follows that $p_{1}, \ldots, p_{n-1}, \infty$ are complete regular ends. Moreover, they are embedded ends since $\operatorname{deg} G+\operatorname{deg} G_{*}(=1+(n-1)=n)$ is equal to the number of ends (see Theorem 3.13).

Finally, we give examples of a complete flat front of genus 1 . 

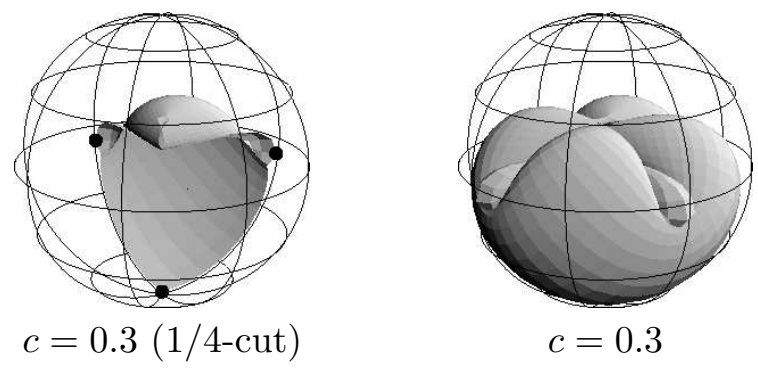

$$
c=0.3
$$

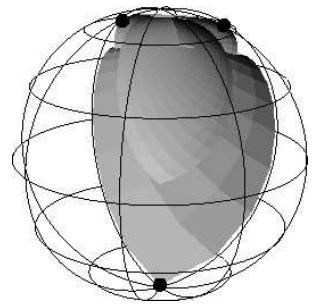

$$
c=1.0(1 / 4 \text {-cut })
$$

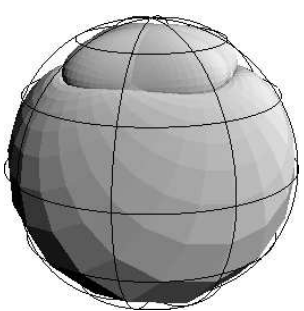

$$
c=1.0
$$

Figure 4. Genus-1 flat fronts with 5 embedded ends (Example 4.6). In the figures of $1 / 4$-cut (left), the ends are shown as the dotted points.

Example 4.6 (flat front of genus 1 with 5 embedded ends). Let $\wp$ denote the Weierstrass $\wp$ function on the square torus $T^{2}=\mathbf{C} /\{\mathbf{Z} \oplus i \mathbf{Z}\}$. We note that $\wp$ satisfies the differential equation

$$
\left(\wp^{\prime}\right)^{2}=4 \wp\left(\wp^{2}-e_{1}^{2}\right), \quad e_{1}=\wp(1 / 2) .
$$

Take the meromorphic functions

$$
G=\wp^{\prime}, \quad G_{*}=-\frac{8 e_{1}^{2}}{3} \frac{\wp}{\wp^{\prime}}
$$

on $T^{2}$. Let $M^{2}$ be a Riemann surface $T^{2}$ punctured at 5 points where $G$ and $G_{*}$ take the same value:

$$
M^{2}:=T^{2} \backslash\left\{z ; \wp(z)\left(3 \wp(z)^{2}-e_{1}^{2}\right)=0\right\} .
$$

We remark that $\wp$ has a double zero at $z=(1+i) / 2$, and $3 \wp^{2}-e_{1}^{2}$ has 4 simple zeros.

For these data, a computation gives

$$
\frac{d G}{G-G_{*}}=\frac{3}{2} \frac{\wp^{\prime}}{\wp} d z
$$

This implies that conditions (1) and (2) of Theorem 2.11 are satisfied. Therefore, the Riemann surface (4.10) and the meromorphic functions (4.9) define a flat front. 
The first canonical form $\omega$ and the Hopf differential $Q$ are computed to be

$$
\omega=-\frac{2}{c^{2}} \frac{3 \wp^{2}-e_{1}^{2}}{\wp^{3}} d z, \quad Q=\frac{-6 e_{1}^{2}\left(\wp^{2}+e_{1}^{2}\right)}{\wp\left(3 \wp^{2}-e_{1}^{2}\right)} d z^{2},
$$

from which the completeness of the ends $\left\{z ; \wp\left(3 \wp^{2}-e_{1}^{2}\right)=0\right\}$ follows. One can also verify the consistency of the data $G, \omega$ and $Q$ by formula (1.10) in [7]. Obviously, all ends are regular.

Since $G_{*}$ has only simple zeros, at $z=0$ and $\frac{1}{2}(1+i)$, its degree is 2. Clearly $\operatorname{deg} G=3$. Hence, the equality in Theorem 3.13 is attained. Therefore all 5 ends are embedded.

\section{References}

[1] R. Bryant, Surfaces of mean curvature one in hyperbolic space, in Théorie des variétés minimales et applications, Astérisque, 154-155 (1988), 321-347, MR 0955072, Zbl 0635.53047.

[2] S. Cohn-Vossen, Kürzeste Wege und Totalkrümmung auf Flächen, Composito Math., 2 (1935), 69-133, Zbl 0011.22501.

[3] Y. Fang, The Minding formula and its applications, Arch. Math., 72(6) (1999), 473480, MR 1683219, Zbl 0958.53042.

[4] J.A. Gálvez, A. Martínez and F. Milán, Flat surfaces in hyperbolic 3-space, Math. Ann., 316 (2000), 419-435, MR 1752778, Zbl 1003.53047.

[5] L.P.M. Jorge and W. Meeks III, The topology of complete minimal surfaces of finite total Gaussian curvature, Topology, 22 (1983), 203-221, MR 0683761, Zbl 0517.53008.

[6] M. Kokubu, W. Rossman, K. Saji, M. Umehara and K. Yamada, Singularities of flat fronts in hyperbolic 3-space, preprint, math.DG/0401110.

[7] M. Kokubu, M. Umehara and K. Yamada, An elementary proof of Small's formula for null curves in $\operatorname{PSL}(2, \mathbf{C})$ and an analogue for Legendrian curves in $\operatorname{PSL}(2, \mathbf{C})$, Osaka J. Math., 40(3) (2003), 697-715, MR 2003744.

[8] R. Osserman, Global properties of minimal surfaces in $E^{3}$ and $E^{n}$, Ann. of Math., 80 (1964), 340-364, MR 0179701, Zbl 0134.38502.

[9] S. Sasaki, On the differential geometry of tangent bundles of Riemannian manifolds, Tôhoku Math. J., 10 (1958), 338-354, MR 0112152, Zbl 0086.15003.

[10] S. Sasaki, On the differential geometry of tangent bundles of Riemannian manifolds II, Tôhoku Math. J., 14 (1962), 146-155, MR 0145456, Zbl 0109.40505.

[11] K. Shiohama, Total curvatures and minimal areas of complete open surfaces, Proc. Amer. Math. Soc., 94 (1985), 310-316, MR 0784184, Zbl 0582.53036.

[12] M. Umehara and K. Yamada, A duality on CMC-1 surfaces in hyperbolic space and a hyperbolic analogue of the Osserman inequality, Tsukuba J. Math., 21(1) (1997), 229-237, MR 1467234, Zbl 1027.53010.

Received January 21, 2003 and revised September 4, 2003. 
Department of Natural Science

SCHOOL OF ENGINEERING

TOKYo Denki University

Chiyoda-Ku, TOKYo, 101-8457

JAPAN

E-mail address: kokubu@cck.dendai.ac.jp

Department of Mathematics

Graduate School of Science

OSAKA UNIVERSITY

TOYONAKA, OSAKa 560-0043

JAPAN

E-mail address: umehara@math.wani.osaka-u.ac.jp

Faculty of Mathematics

KYUSHU UNIVERSITY

Higashi-Ku, FukuOKa 812-8581

JAPAN

E-mail address: kotaro@math.kyushu-u.ac.jp 